\title{
Publisher's Note: Test of non-Newtonian gravitational forces at micrometer range with two-dimensional force mapping [Phys. Rev. D 94, 122005 (2016)]
}

Jianbo Wang, Shengguo Guan, Kai Chen, Wenjie Wu, Zhaoyang Tian, Pengshun Luo, Aizi Jin, Shanqing Yang, Chenggang Shao, and Jun Luo

Q (Received 14 May 2018; published 29 May 2018)

DOI: 10.1103/PhysRevD.97.109901

This paper was published online on 22 December 2016 with an error in Eq. (6). Equation (6) should read as

$$
P_{i j}\left(F_{i j}^{\exp } \mid x_{00}, \alpha, \lambda\right)=\frac{1}{\sqrt{2 \pi} \sigma_{i j}} e^{-} \frac{\left(F_{i j}^{\exp }-F_{i j}^{T}\right)^{2}}{2 \sigma_{i j}^{2}} .
$$

The equation has been corrected as of 22 May 2018. The equation is incorrect in the printed version of the journal. 